\title{
Polarization-resolved second-harmonic-generation optical coherence tomography in collagen
}

\author{
Brian E. Applegate and Changhuei Yang \\ Department of Biomedical Engineering, Duke University, Durham, North Carolina 27708 \\ Andrew M. Rollins \\ Department of Biomedical Engineering, Case Western Reserve University, Cleveland, Ohio 44106 \\ Joseph A. Izatt \\ Department of Biomedical Engineering, Duke University, Durham, North Carolina 27708
}

Received April 27, 2004

\begin{abstract}
We describe a novel imaging technique, second-harmonic-generation optical coherence tomography (SHOCT). This technique combines the spatial resolution and depth penetration of optical coherence tomography (OCT) with the molecular sensitivity of second-harmonic-generation spectroscopy. As a consequence of the coherent detection required for OCT, polarization-resolved images arise naturally. We demonstrate this new technique on a skin sample from the belly of Icelandic salmon, acquiring polarization-resolved SHOCT and OCT images simultaneously. (c) 2004 Optical Society of America

OCIS codes: $110.4500,190.4160,190.4710$.
\end{abstract}

Optical coherence tomography (OCT) is a noninvasive optical imaging technique that exploits the scattering properties of tissue to provide tomographic sectioning of subsurface tissue morphology. OCT is rapidly evolving with the development of ultrahighresolution $^{1}\left(<5 \mu \mathrm{m}\right.$ axially) and high-speed ${ }^{2}$ real-time imaging systems. The significance of OCT would be greatly enhanced by the development of a capability to measure molecular specific signals. Similar to confocal microscopy, OCT is not inherently sensitive to molecular species. However, as confocal microscopy has been revolutionized by the introduction of fluorescence microscopy, two-photon emission microscopy, and second-harmonic-generation microscopy, similar molecular-sensitive imaging modalities would greatly enhance the utility of OCT. Unfortunately, observation of fluorescence, the strongest of the molecular processes exploited in microscopy, is not possible because of the coherent detection method employed in OCT. Only molecular processes that result in coherent emission of light may be detected.

As the first demonstration of molecular contrast OCT, Rao et al. ${ }^{3}$ adapted pump-probe spectroscopy to optical coherent tomography (PPOCT) and observed transient absorption of a triplet state of the dye Methylene Blue. Although PPOCT has the potential to be used with a number of different contrast agents, its major failing is that it requires intimate knowledge of the excited electronic state manifold of the molecular contrast agent. This type of information is typically lacking.

Second-harmonic generation is a coherent process and can thus be directly detected in OCT. In contrast to PPOCT, the contrast agents that are used in the confocal analog, second-harmonic imaging microscopy, are suitable for use with second-harmonic-generation optical coherence tomography (SHOCT) without further knowledge of the excited states of the contrast agents. Additional advantages shared with the secondharmonic imaging microscopy technique are that, since second-harmonic generation is a nonresonant process, the effects of photobleaching and photodamage to tissue are at least in principle negligible.

The physical origins of second-harmonic generation as well as their implications for the imaging of tissue morphology are well known (see, e.g., Refs. 4 and 5). The power in the second harmonic, $P_{2 \omega}$, is proportional to the square of the power in the fundamental, $P_{1 \omega}$. In the electric dipole approximation, second-harmonic generation can occur only in a noncentrosymmetric medium. In biological specimens it is therefore observed only at interfaces such as cell membranes or in highly ordered structures, including those typically formed by aligned collagen fibers. One may determine the orientation of the molecules exhibiting a second-harmonic response by resolving the polarization of the second-harmonic signal into components parallel and perpendicular to the incident field polarization. This information is typically quantified in terms of the anisotropy parameter, which for linearly polarized light is given by $\beta=\left(I_{\|}-I_{\perp}\right) /\left(I_{\|}+2 I_{\perp}\right)$, where $I_{\|}$and $I_{\perp}$ are the second-harmonic signal intensities with polarization parallel and perpendicular, respectively, to the incident field polarization. ${ }^{6}$

The equation for the signal-to-noise ratio (SNR) of an OCT system operating in the ideal shot-noise limit was derived previously ${ }^{7}$ and is given by $\eta P_{s} R_{s} /(2 \hbar \nu B)$, where $\eta$ is the quantum efficiency of the receiver, $P_{s}$ is the sample signal power, $R_{s}$ is the sample reflectance, $\hbar$ is Planck's constant, $\nu$ is the center frequency of the radiation, and $B$ is the bandwidth of the detection electronics. For SHOCT, $P_{s}$ is equal to $P_{2 \omega}$ and $R_{s}$ is equal to $R_{2 \omega}$, hence

(C) 2004 Optical Society of America 


$$
\mathrm{SNR}_{2 \omega}=\frac{\eta P_{2 \omega} R_{2 \omega}}{2 h \nu B}=\frac{\eta a P_{1 \omega}{ }^{2} R_{2 \omega}}{2 h \nu B}
$$

where we define the proportionality constant $a$ by the equation $P_{2 \omega}=a P_{1 \omega}^{2}$ and $R_{2 \omega}$ incorporates both the reflectivity of the sample at the frequency of the second harmonic and the probability of second-harmonic generation in the backward direction.

Using Eq. (1), we can recast $\beta$ in a more useful form. Since both polarizations are measured with the same focusing of the incident laser, Eq. (1) may be rewritten as

$$
\beta=\frac{\mathrm{SNR}_{2 \omega, \|}-\mathrm{SNR}_{2 \omega, \perp}}{\mathrm{SNR}_{2 \omega, \|}+2 \mathrm{SNR}_{2 \omega, \perp}} .
$$

It should be noted that, since it is impossible in this experiment to illuminate only a single molecule with the incident laser, the anisotropy parameter is actually an average over the focal area.

The optical setup for SHOCT is only slightly modified from that utilized for a standard bulk optic time-domain OCT system. Figure 1(a) is a schematic of the SHOCT setup. The light source was a femtosecond Nd:glass laser (100 fs, $52 \mathrm{MHz}, 170 \mathrm{~mW}$ ) operating at a center wavelength of $1059 \mathrm{~nm}(15-\mathrm{nm}$ bandwidth). The second-harmonic light in the reference arm was generated by a KDP crystal. A $\lambda / 4$ wave plate was placed after the KDP crystal and adjusted so that the reference-arm light was linearly polarized at $45^{\circ}$. This allowed us to coherently detect both linear orthogonal polarization states. The sample-arm objective provided an effective numerical aperture of $\sim 0.025$, a spot size of $\sim 28 \mu \mathrm{m}$, and a depth of focus of $\sim 1 \mathrm{~mm}$. In the detection arm of the interferometer a dichroic mirror was used to separate the fundamental and the second harmonic. The second harmonic was further separated into orthogonal linear polarization states by a polarizing beam splitter. Photodiode detectors were used for both the fundamental (InGaAs, New Focus) and the second-harmonic (Si, New Focus) light. The interferometric signal was demodulated with a pair of lock-in amplifiers before being passed to a PC/DAQ card. Because of physical limitations of the DAQ card, the signal from only two detectors could be recorded simultaneously. Axial scanning (A-scan) was accomplished by use of a corner prism attached to a scanning galvanometer, oscillating at approximately $5 \mathrm{~Hz}$, as the reference-arm mirror. We accomplished lateral scanning (B-scan) by stepping a computer-controlled translation stage at the desired step size and rate.

The system was characterized with a BBO crystal placed in the sample arm to generate secondharmonic light. Figure 1(b) shows a section of the interferograms resulting from a mirror in the sample arm, due to both the fundamental and the second harmonic, recorded by sending the signal from the detectors directly into the DAQ card. As expected, the frequency of the second-harmonic interferogram was twice that of the fundamental. The inset in Fig. 1(b) shows the full interferogram for both the fundamental and the second harmonic plotted with an artificial offset in space so that the full envelope may be seen for both. Because of the dispersion mismatch between the fundamental and the second-harmonic wavelengths the signals from both are separated in space. The interferograms in Fig. 1(b) were therefore manually overlapped.

Figure 1(c) is a plot of the measured SNR versus $P_{1 \omega}$ for the second harmonic and the fundamental. As expected, the experimental points for the fundamental form a line that passes through the origin. Likewise, from Eq. (1) we expect the second-harmonic SNR to have a quadratic dependence on $P_{1 \omega}$. The theoretical points are based on Eq. (1), using a measured value for the second-harmonic conversion efficiency $\left(a=1.09 \times 10^{-3} \mathrm{~W}^{-1}\right)$ of the sample-arm BBO crystal. The line through the second-harmonic experimental points is a fit of the data to Eq. (1) with one additional parameter to account for system losses. The measured SNR at the sample-arm power used for imaging $(\sim 70 \mathrm{~mW})$ was $114 \mathrm{~dB}$ for the fundamental and $113 \mathrm{~dB}$ (in the limit of perfect conversion efficiency, i.e., $a=1$ ) for the second harmonic, which compares to the theoretical shot-noise limit of 131 and $129 \mathrm{~dB}$, respectively. Although the SNRs for the fundamental and second harmonic are nearly equivalent in the limit of $a=1$, for real samples $a<<1$, resulting in significantly weaker SNR for SHOCT. Limitations to the current setup prevented us from simultaneously optimizing the SNRs of both the fundamental and the second harmonic, hence the system was optimized for the second harmonic during imaging.

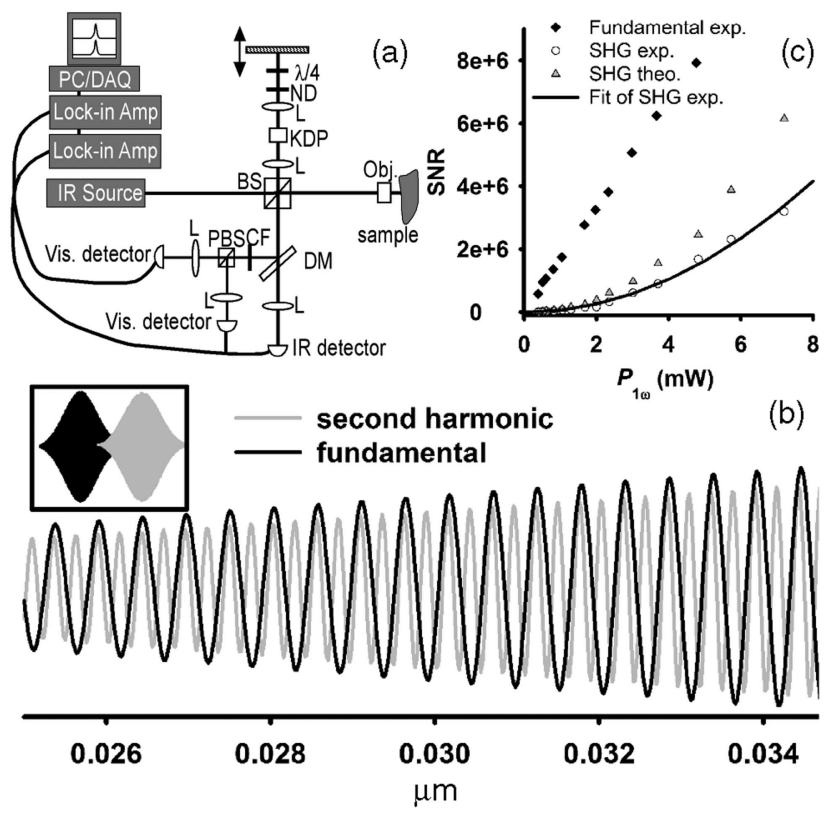

Fig. 1. (a) Schematic of the optical system used for SHOCT: BS, cube beam splitter; Obj., objective lens; L's, lenses; KDP, potassium dihydrogen phosphate crystal; $\mathrm{ND}$, neutral-density filter; DM, dichroic mirror; CF, color filter; PBS, polarizing cube beam splitter. (b) Section of the interferograms formed by the fundamental and the second harmonic. The inset shows the full interferometric signal for both, with an arbitrary offset. (c) SNR plotted as a function of the fundamental power, $P_{1 \omega}$. The measured SNR of the second-harmonic signal has a quadratic dependence on $P_{1 \omega}$ as predicted by Eq. (1). 


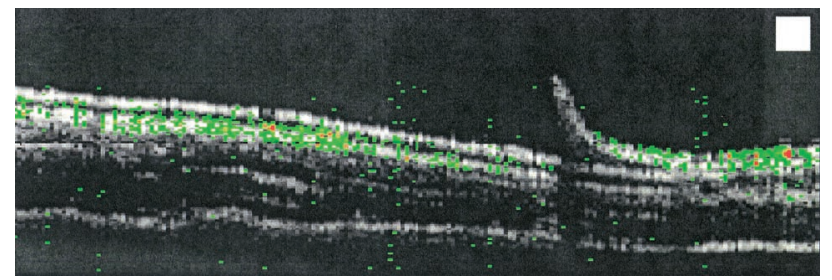

Fig. 2. Overlay of the SHOCT image (green-red) on the fundamental OCT image acquired simultaneously over $\sim 40 \mathrm{~min}$. The scale bar in the upper right corner is $250 \mu \mathrm{m} \times 250 \mu \mathrm{m}$.
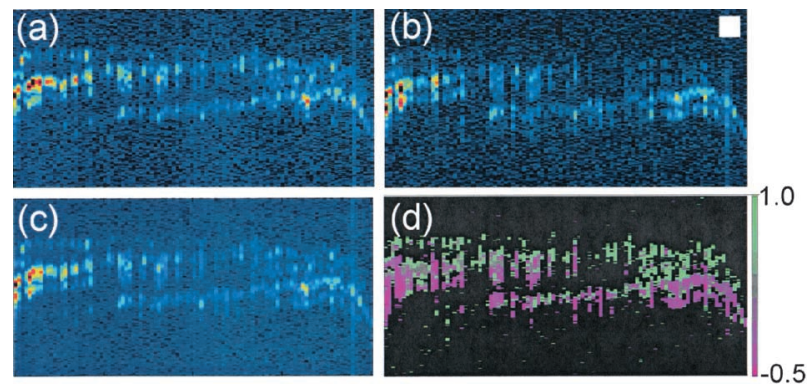

Fig. 3. (a) SHOCT image of the overlap of three fish scales, recorded with the reference-arm secondharmonic light polarization parallel to the fundamental light polarization. (b) SHOCT image recorded with the reference-arm second-harmonic light polarization perpendicular to the fundamental light polarization. (c) Polarization-independent image derived from (a) and (b). (d) Image of the anisotropy parameter, $\beta$. The color scale varies from magenta for -0.5 to green for 1.0 . The black space is a result of thresholding out the areas with no signal, defined as having a SNR of $\leq 3.0$. The parallel and perpendicular images were acquired simultaneously over $\sim 35 \mathrm{~min}$. The scale bar in the upper right corner is $125 \mu \mathrm{m} \times 125 \mu \mathrm{m}$.

To demonstrate this technique in a biological sample, we imaged skin samples from the belly of an Icelandic salmon. We expected to observe strong second-harmonic generation from the salmon scales because of the high concentration of highly ordered collagen. Although the underlying dermal layers do contain collagen, they are not as highly ordered as in the scales. Figure 2 is an overlay of the SHOCT image on the fundamental OCT image acquired simultaneously. The background from the SHOCT image was filtered by thresholding, and the image was rendered on a false-color scale ranging from green to red. The SHOCT image is dominated by the signals from two overlapping scales, with only a weak hint of the underlying dermal layers predominantly on the right-hand side of the image. The slight curling of the rightmost scale was due to drying of the sample during imaging. We averaged $100 \mathrm{~A}$-scans (axial line scans) and recorded every $50 \mu \mathrm{m}$ to build up this $6 \mathrm{~mm}$ (lateral) by $2 \mathrm{~mm}$ (axial) B-scan.

We gleaned further structural information from the SHOCT image by recording signals from both parallel and perpendicular polarizations. The complete polarization-resolved SHOCT image of a second skin sample is shown in Figs. 3(a) and 3(b). This $2 \mathrm{~mm}$ (lateral) by $1 \mathrm{~mm}$ (axial) B-scan showing the overlap of three scales was recorded by averaging $100 \mathrm{~A}$-scans separated by $20 \mu \mathrm{m}$. The polarization-independent image derived from Figs. 3(a) and 3(b) is given in Fig. 3(c) for comparison. It is evident from this image that we were able to image completely through the three layers of highly scattering scale. Using the average refractive index of hydrated type I collagen ${ }^{8}$ (1.43), we found that the penetration depth was at least $280-350 \mu \mathrm{m}$.

Close inspection of the polarization-resolved images reveals distinct differences between the two. These differences are rooted in the orientation of the collagen fibers relative to the incident laser polarization. As noted above, this may be quantified by the anisotropy parameter, $\beta$. Applying Eq. (2) to Figs. 3(a) and 3(b) results in Fig. 3(d). The black space in Fig. 3(d) is a result of thresholding out the background, which was considered to be any signal with a SNR of $<3.0$. The patches of pixels in Fig. 3(d) with similar color indicate collagen domains where all the fibers have a similar orientation.

In conclusion, we have developed a new technique based on the combination of second-harmonic-generation spectroscopy with optical coherence tomography. Polarization-resolved second-harmonic images arise naturally as a result of the coherent detection technique used in SHOCT. SHOCT provides information from molecular-specific and polarization-resolved signals that is complimentary to the tomographic information provided by OCT. For the salmon skin samples studied in this work the SHOCT image identifies the structures with large collagen content and the relative orientation of the collagen.

We acknowledge funding of this work through grant R24 EB000243 from the National Institutes of Health. B. E. Applegate (brian.applegate@duke.edu) acknowledges fellowship support through the National Institutes of Health (T32 EB001630).

\section{References}

1. W. Drexler, J. Biomed. Opt. 9, 47 (2004).

2. A. M. Rollins, M. D. Kulkarni, S. Yazdanfar, R. Ung-Arunyawee, and J. A. Izatt, Opt. Express 3, 219 (1998), http://www.opticsexpress.org.

3. K. D. Rao, M. A. Choma, S. Yazdanfar, A. M. Rollins, and J. A. Izatt, Opt. Lett. 28, 340 (2003).

4. P. J. Campagnola and L. M. Loew, Nat. Biotechnol. 21, 1356 (2003).

5. Y. R. Shen, Nonlinear Spectroscopy for Molecular Structure Determination, R. W. Field, E. Hirota, J. P. Maier, and S. Tsuchiya, eds. (Blackwell Science, Malden, Mass., 1998), pp. 249-271.

6. W. Mohler, A. C. Milliard, and P. J. Campagnola, Methods 29, 97 (2003).

7. E. A. Swanson, D. Huang, M. R. Hee, J. G. Fujimoto, C. P. Lin, and C. A. Puliafito, Opt. Lett. 17, 151 (1992).

8. X.-J. Wang, T. E. Milner, M. C. Chang, and J. S. Nelson, J. Biomed. Opt. 1, 212 (1996). 